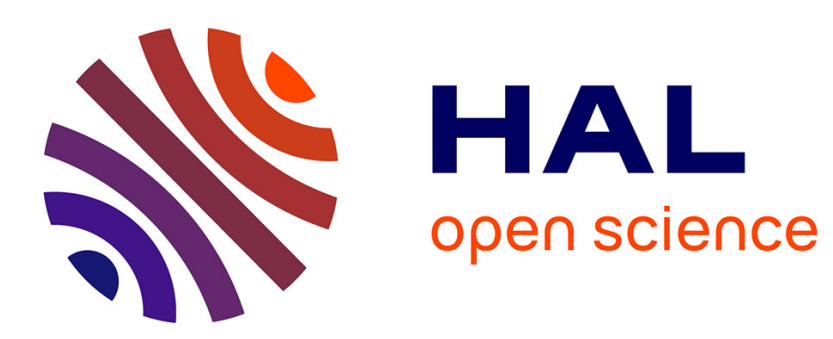

\title{
Tacit collusion in a one-shot game of price competition with soft capacity constraints
}

\author{
Marie-Laure Cabon-Dhersin, Nicolas Drouhin
}

\section{To cite this version:}

Marie-Laure Cabon-Dhersin, Nicolas Drouhin. Tacit collusion in a one-shot game of price competition with soft capacity constraints. 2012. hal-00709093v2

\section{HAL Id: hal-00709093 \\ https://hal.science/hal-00709093v2}

Preprint submitted on 4 Oct 2012

HAL is a multi-disciplinary open access archive for the deposit and dissemination of scientific research documents, whether they are published or not. The documents may come from teaching and research institutions in France or abroad, or from public or private research centers.
L'archive ouverte pluridisciplinaire HAL, est destinée au dépôt et à la diffusion de documents scientifiques de niveau recherche, publiés ou non, émanant des établissements d'enseignement et de recherche français ou étrangers, des laboratoires publics ou privés. 


\title{
Tacit collusion in a one-shot game of price
}

\section{competition with soft capacity constraints*}

\author{
Marie-Laure Cabon-Dhersin` Nicolas Drouhin ${ }^{\ddagger}$
}

September 26, 2012

\begin{abstract}
This paper analyses price competition in the case of two firms operating under constant returns to scale with more than one production factor. Factors are chosen sequentially in a two-stage game generating a soft capacity constraint and implying a convex short term cost function in the second stage of the game. We show that tacit collusion is the only predictable result of the whole game i.e. the unique payoff-dominant pure strategy Nash equilibrium. Technically, this paper bridges the capacity constraint literature on price competition and that of the convex cost function.
\end{abstract}

Key words: price competition, tacit collusion, convex cost, capacity constraint

Code JEL: L13, D43

\footnotetext{
${ }^{*}$ We thank for their helpful comments and suggestions, the editors, two anonymous referees, various seminar participants, and Simon Anderson, Francis Bloch, Raymond Deneckere, Joseph Harrigton, Edi Karni, Bertrand Munier, André de Palma. We thank also Alain Goergen for helping us to simulate some special cases on Mapple.

†Université de Rouen, CREAM, Adress: 3 avenue Pasteur, 76100 Rouen, France. (marielaure.cabon-dhersin@univ-rouen.fr)

${ }^{\ddagger}$ Corresponding author at Ecole Normale Supérieure de Cachan and CES -Université de Paris 1Panthéon Sorbonne, Adress: Ens Cachan, 61 avenue du Président Wilson, 94230 Cachan, France. (drouhin@ecogest.ens-cachan.fr)
} 


\section{Introduction.}

The literature on Industrial Organization emphasizes the role of threats and retaliations in a dynamic game framework to explain tacit collusion (Friedman, 1971; Abreu, 1986; Benoit and Krishna, 1987; Feuerstein, 2005). This paper gives an example of a market in which the collusive outcome arises as a predictable result of a non-repeated price competition duopoly with two stages. This is consistent with the experimental findings of a remarkable degree of coordination around a collusive price by two firms (Abbink and Brandts, 2008). Following Ivaldi et al. (2003), tacit collusion needs not to involve any collusion in the legal sense, and, in particular, no communication between the parties. "It is referred to as tacit collusion only because the outcome (in terms of prices set or quantities produced, for example) may well resemble that of explicit collusion or even of an official cartel".

We start from the Bertrand competition model initiated by Dastidar (1995) ${ }^{1}$ and extended recently by Baye and Morgan (2002); Novshek and Chowdhury (2003); Hoernig (2007); Bagh (2010). In this setting, firms face convex costs and are committed to satisfying the full demand. By lowering its price, a firm increases its revenue by higher sales. But, costs being convex, they will increase even more, making this deviation non-profitable. For this reason, a continuum of prices above the competitive price can be sustained as Nash equilibria in pure strategies ${ }^{2}$, inducing a coordination problem in a non-repeated framework. To claim the possibility of tacit collusion in this framework, two questions still need to be answered: 1) Is the collusive outcome within the set of the Nash equilibria? 2) Is there a plausible selection procedure to achieve the collusive outcome as the unique solution? Dastidar (2001) answers the first question, but only in the symmetric case. As far as we know, the second question has not been treated explicitly.

According to Harsanyi and Selten (1988), two criteria can be used to solve the coordination issue: The payoff dominance criterion which appears to be the natural 
criterion when it is common knowledge that both players are fully rational ${ }^{3}$, and the risk dominance criterion that can be invoked when payoff dominance is insufficient to provide uniqueness. Unfortunately, risk dominance may not be applied in the context of Bertrand competition with convex costs, because of the infinity of equilibria.

In this paper, we will add a sequential choice of production factors into Dastidar's (1995) approach of Bertrand competition. As we will demonstrate, this plays a key role in the coordination mechanism that leads to tacit collusion. Our model starts from a constant returns to scale production function with two substitutable production factors chosen sequentially. In the first stage, the firms invest i.e. they choose the quantity of the fixed factors, quantity that will be invariable over the second stage. In the second stage, firms compete on price and determine the quantity of variable factors needed to satisfy the demand they will face. This implies that, when the first factor is fixed, the short run marginal cost is convex in the second stage ${ }^{4}$. The sequential choice of the production factors is certainly the central hypothesis of our approach. On the one hand, it is a standard hypothesis in economic analysis, a textbook case renewing the Marshallian tradition of distinguishing between short and long term cost functions. On the other hand, as far as we know, it appears that it has not been used in the recent literature about price competition. However, we want to point out that this assumption is a natural generalization of the notion of capacity constraints initiated by Edgeworth (1925). There is a long tradition in Industrial Organization of considering firms that are capacity constrained (Vives, 1980; Kreps and Scheinkman, 1983; Allen and Hellwig, 1986; Davidson and Deneckere, 1986; Allen et al., 2000). In those models, the constraint is drastic (i.e. it is impossible to produce above the capacity). In our model, the choice of the fixed factor corresponds to the choice of the production capacity. But the usual way to model capacity constraints is equivalent, in our setting, with an assumption of perfect complementarity of fixed and variable factors. In this case, when the capacity of production is binding in the second stage, it is impossible to produce more, whatever the quantity of variable factor. Our hypothesis of substitutability between fixed and 
variable factors introduces a less drastic (soft) notion of capacity constraints ${ }^{5}$, and in many cases a more realistic one. For example, we can take, as an illustration, the retailing sector. The fixed factor refers to the surface needed to sell and the length of the shelves used to display the products, whereas the variable factor can be interpreted as the number of employees needed to fill up the shelves. For a given surface of the store, there exists an optimal number of employees minimizing the long-term average cost. But if demand is superior to the output corresponding to this optimal number, the shelves will be emptied more quickly, and you will need more employees to fill up the shelves continuously and to satisfy this supplementary demand.

In this paper, we will show that the standard selection criterion of payoff dominance is not sufficient to assure uniqueness of equilibrium. But if we add our notion of soft capacity constraint, the uniqueness is guaranteed in the two-stage game and the equilibrium chosen is a collusive one.

The paper is organized as follows. Section 2 presents the model. Reasoning backwards, the second stage of the game is solved in Section 3 and the first one in Section 4. The final section concludes.

\section{The model.}

There are two identical firms in a market for a homogeneous good. Consider a twostage game where firms invest in the first stage and simultaneously choose the price in the second stage. We introduce the following assumptions:

1. Firms rely on a technology represented by a two-factor constant returns to scale production function. The first factor is fixed in the second stage, while the second one varies to satisfy the demand faced by the firm ${ }^{6}$. For the firm $i$, the fixed factor will be denoted $z_{i}$ and the variable one, $v_{i}$. The fixed factor price is $w_{1}$, and the variable one is $w_{2}$. For readability, we will use a Cobb-Douglas production function $y_{i}=a z_{i}^{\alpha} v_{i}^{1-\alpha}$ with $i=1,2$ and $i \neq j$, where $a$ is a positive 
constant, and $\alpha$, the elasticity of the production according to the level of the fixed factor, is a constant between 0 and 1 . The total cost function can be written.

$$
C\left(y_{i} ; z_{i} ; v_{i}\right)=w_{1} z_{i}+w_{2} v_{i}=w_{1} z_{i}+w_{2} \frac{y_{i}^{\frac{1}{1-\alpha}}}{a^{\frac{1}{1-\alpha}} z_{i}^{\frac{\alpha}{1-\alpha}}}
$$

Viewed from the second stage, the first term of the righthand side is sunk and the second term is the short-run cost function. It is continuous, increasing and convex.

2. The demand is continuous, twice differentiable and decreasing.

$D: \mathbb{R}^{+} \longrightarrow \mathbb{R}^{+}$with $D\left(p^{\max }\right)=0, D(0)=Q^{\max }$. Classically, we denote the price elasticity of demand: $\mathcal{E}(p)=p \frac{D^{\prime}(p)}{D(p)}$.

3. Firms have to supply all the demand they face. The demand function is defined as follows:

$$
D_{i}\left(p_{i} ; p_{j}\right)= \begin{cases}0 & \text { if } p_{i}>p_{j} \\ \frac{1}{2} D\left(p_{i}\right) & \text { if } p_{i}=p_{j} \\ D\left(p_{i}\right) & \text { if } p_{i}<p_{j}\end{cases}
$$

We can now express the profit $\pi_{i}$ for each firm $i$.

$$
\begin{aligned}
& \pi_{i}\left(p_{i}, p_{j}, z_{i}\right)=p D_{i}\left(p_{i}, p_{j}\right)-C_{i}\left(D_{i}\left(p_{i}, p_{j}\right), z_{i}\right) \\
& \pi_{i}\left(p_{i}, p_{j}, z_{i}\right)= \begin{cases}-w_{1} z_{i} & \text { if } p_{i}>p_{j} \\
p \frac{D(p)}{2}-w_{1} z_{i}-w_{2} \frac{\left(\frac{D(p)}{2}\right)^{\frac{1}{1-\alpha}} a^{\frac{1}{1-\alpha}} z_{i} \frac{\alpha}{1-\alpha}}{=} \hat{\pi}\left(p, z_{i}\right) & \text { if } p_{i}=p_{j}=p \\
p D(p)-w_{1} z_{i}-w_{2} \frac{D(p)^{\frac{1}{1-\alpha}}}{a^{\frac{1}{1-\alpha}} z_{i} \frac{\alpha}{1-\alpha}} \stackrel{\text { def }}{=} \pi\left(p, z_{i}\right) & \text { if } p_{j}>p_{i}=p\end{cases}
\end{aligned}
$$

The function $\hat{\pi}\left(p, z_{i}\right)$ represents the profit of the firm $i$ when both firms quote the same price and the function $\pi\left(p, z_{i}\right)$, represents the profit of firm $i$ when it quotes the lowest price and supplies the market alone. 
4. The demand is such that $\hat{\pi}\left(p, z_{i}\right)$ and $\pi\left(p, z_{i}\right)$ are strictly concave in $p$ and strictly concave in $z, i . \quad$ e. $\partial^{2} \hat{\pi}(p, z) / \partial p^{2}<0, \partial^{2} \hat{\pi}(p, z) \partial z^{2}<0, \partial^{2} \pi(p, z) / \partial p^{2}<0$, $\partial^{2} \pi(p, z) \partial z^{2}$. After trivial calculations, it can be shown that $\partial^{2} \hat{\pi}(p, z) / \partial p \partial z<0$.

We define $\bar{p}_{i}\left(z_{i}\right)$ that solves $\hat{\pi}\left(p, z_{i}\right)=\pi\left(p, z_{i}\right)$. Thus $\bar{p}_{i}\left(z_{i}\right)$ must be interpreted as the critical price for which the firm is indifferent between operating in the market alone or with its competitor. After calculation, we obtain

$$
\bar{p}_{i}\left(z_{i}\right)=\frac{w_{2}}{a^{\frac{1}{1-\alpha}} z_{i}^{\frac{\alpha}{1-\alpha}}}\left(\frac{D\left(\bar{p}_{i}\right)}{2}\right)^{\frac{\alpha}{1-\alpha}}\left(2^{\frac{1}{1-\alpha}}-1\right)
$$

In the second stage, the fixed cost, $w_{1} z_{i}$, is sunk, and the firm will quote a price only if the variable part of the profit is positive i. e. $\hat{\pi}\left(p, z_{i}\right) \geq-w_{1} z_{i}$. Thus, we also define $\hat{p}_{i}$, that solves $\hat{\pi}\left(p, z_{i}\right)=-w_{1} z_{i}$ for a given $z_{i}$, the minimum price compatible with a decision to produce in the second stage.

$$
\hat{p}_{i}\left(z_{i}\right)=\frac{w_{2}}{a^{\frac{1}{1-\alpha}} z_{i}^{\frac{\alpha}{1-\alpha}}}\left(\frac{D\left(\hat{p}_{i}\right)}{2}\right) \frac{\alpha}{1-\alpha}
$$

Finally, we define $p_{i}^{*}$, the price that maximises the profit of firm $i$ when both firms operate in the market. As a shortcut, this price can be interpreted as the cartel price when both firms have chosen the same level of fixed factors in the first stage.

$$
p_{i}^{*}\left(z_{i}\right) \stackrel{\text { def }}{=} \underset{p}{\arg \max }\left\{\hat{\pi}\left(p, z_{i}\right)\right\}=\frac{1}{1+\frac{1}{\mathcal{E}\left(p_{i}^{*}\right)}} \frac{1}{1-\alpha} \frac{w_{2}}{a^{\frac{1}{1-\alpha}} z_{i}^{\frac{\alpha}{1-\alpha}}}\left(\frac{D\left(p_{i}^{*}\right)}{2}\right) \frac{\alpha}{1-\alpha}
$$

Let us note that, for a given $z_{i}, p^{*}\left(z_{i}\right)$ is different from $p^{m}\left(z_{i}\right)$, the monopoly price which maximises the profit of a firm alone in the market.

In the rest of the paper, when reasoning with a given $z_{i}$, we will denote $\hat{\pi}\left(p, z_{i}\right)=$ $\hat{\pi}_{i}(p)$ and $\pi\left(p, z_{i}\right)=\pi_{i}(p)$.

It is important to understand how those profit functions $\hat{\pi}\left(p, z_{i}\right)$ and $\pi\left(p, z_{i}\right)$ and those prices $\hat{p}, p^{*}$ and $\bar{p}$ are organized together. 
Lemma 1 (Geometry of profit functions for a given $z_{i}$ ).

$$
\begin{aligned}
& \hat{p}_{i}<\bar{p}_{i} \\
& \forall p \geqslant \hat{p}_{i}, \hat{\pi}_{i}(p) \geqslant-w_{1} z_{i} \\
& \forall p \in\left[\hat{p}_{i} ; \bar{p}_{i}\right], \forall \mu \in(0, p], \hat{\pi}_{i}(p) \geqslant \pi_{i}(p)>\pi_{i}(p-\mu) \\
& z_{i}>z_{j} \Rightarrow\left\{\begin{array}{c}
\hat{p}_{j}>\hat{p}_{i} \\
\bar{p}_{j}>\bar{p}_{i} \\
p_{j}^{*}>p_{i}^{*}
\end{array}\right.
\end{aligned}
$$

Proof: Following equations (1), (2) and (3), (4a),(4b),(4c) are obvious. Taking the total differential of expressions (1), (2) and (3), we can show that $\forall z, d \hat{p}(z) / d z<0$, $d \bar{p}(z) / d z<0$ and $d p^{*}(z) / d z<0$, proving $(4 \mathrm{~d})$.

The prices $\bar{p}(z)$ and $p^{*}(z)$ will play an important role in the resolution of the game and we have to settle the question of their relative position.

Lemma 2 (Comparison between $\bar{p}(z)$ and $p^{*}(z)$ ).

$\forall \alpha \in(0,1), \exists ! \tilde{z}$,

$$
\begin{aligned}
& z=\tilde{z} \Leftrightarrow \bar{p}(z)=p^{*}(z) \equiv \tilde{p} \Leftrightarrow \frac{1}{1-\alpha} \frac{1}{2^{\frac{1}{1-\alpha}}-1}=1+\frac{1}{\mathcal{E}(\tilde{p})} \\
& z<\tilde{z} \Leftrightarrow \bar{p}(z)>p^{*}(z) \Leftrightarrow \frac{1}{1-\alpha} \frac{1}{2^{\frac{1}{1-\alpha}}-1}<1+\frac{1}{\mathcal{E}(\bar{p}(z))}
\end{aligned}
$$

Proof: in the appendix.

$\tilde{z}$ is exogenous, only determined by the special form of the demand and production functions. If the firm chooses a level of $z$ lower than $\tilde{z}$, then $\bar{p}(z)$ will be above $p^{*}(z)$. However, if the firm chooses a higher level, then $\bar{p}(z)$ will be lower than $p^{*}(z)$. So when solving the first stage of the game, $z$ will be endogenous, and we have to keep in mind the qualitative implications for the resolution of the second stage. For a given demand 
function, $\alpha$ is the sole determinant of $\tilde{p}$ position. When $\alpha$ tends to 1 , the condition (5-b) is more easily satisfied.

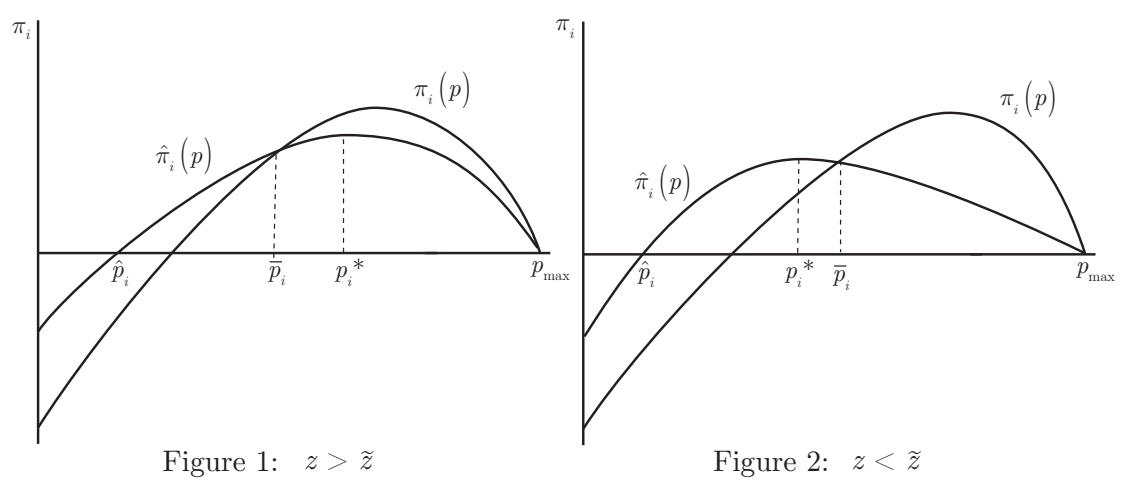

Figure 1 and Figure 2 illustrate the geometry of the profit functions. Considering just one firm with a definite level of the fixed factor, (4a), (4b) and (4c) allow us to draw the functions $\hat{\pi}_{i}(p)$ and $\pi_{i}(p)$.

These functions are parameterized by the level of the fixed factors. What happens if this level increases? (4d) shows that the curves will be transformed with $\hat{p}_{j}, \bar{p}_{j}$ and $p^{*}$ moving to the left.

The two-stage game is solved by backward induction. First, we analyze the price competition in the second stage of the game, for a given fixed input at levels $z_{1}$ and $z_{2}$. Secondly, the firms optimally choose their fixed input levels in the first stage of the game.

\section{The second stage of the game: price competition}

In this section, we take the firms' fixed input levels as given and look for the Nash equilibrium in prices. Thus, to simplify the exposition, we omit the $z$ variable when denoting the price. For reasons that will become clearer when we resolve the first stage of the game in the next section, we will consider the possibility that $z_{1}$ and $z_{2}$, chosen in the first stage, can be different but "not too much". More precisely, we will assume 
that $z_{1}$ and $z_{2}$ are such that $\left[\hat{p}_{1}, \bar{p}_{1}\right] \cap\left[\hat{p}_{2}, \bar{p}_{2}\right] \neq \emptyset$.

\subsection{Nash Equilibria}

Proposition 1. In the second stage, $\left(p_{1}, p_{2}\right)$ is a pure strategy Nash equilibrium if and only if $p_{1}=p_{2}=p^{N}$, with $p^{N} \in\left[\hat{p}_{1}, \bar{p}_{1}\right] \cap\left[\hat{p}_{2}, \bar{p}_{2}\right]=\left[\max \left(\hat{p}_{1}, \hat{p}_{2}\right), \min \left(\bar{p}_{1}, \bar{p}_{2}\right)\right] \neq \emptyset$.

Proof: in the appendix

The Nash equilibrium prediction in the second stage game is basically the one of Dastidar (1995), with the same drawback. With the exception of the very special case $\bar{p}_{i}=\hat{p}_{j}$, in which there is a unique Nash equilibrium, all the other cases are characterized by an infinite number of equilibria, with a minimum zero short-term profit equilibrium price and a maximum above the competitive price.

\subsection{Equilibrium selection}

In this subsection, we will use payoff dominance criterion to reduce the set of equilibria and discuss the uniqueness problem. An equilibrium point is said to be payoff-dominant if it is not strictly dominated by an other equilibrium point, i.e. there exists no other equilibrium in which payoffs are higher for all players.

Proposition 2. In the second stage, $\left(p_{1}, p_{2}\right)$ is a payoff-dominant pure strategy Nash equilibrium if and only if: $p_{1}=p_{2}=p^{P D N}$,

$$
\text { with } p^{P D N} \in \mathrm{I}=\left[\max \left(\hat{p}_{1}, \hat{p}_{2}, \min \left(p_{1}^{*}, p_{2}^{*}, \bar{p}_{1}, \bar{p}_{2}\right)\right), \min \left(\max \left(p_{1}^{*}, p_{2}^{*}\right), \bar{p}_{1}, \bar{p}_{2}\right)\right]
$$

Proof: in the appendix

Proposition 2 reduces the set of predictable outcomes of the game but does not imply uniqueness. However, the following corollaries will prove that uniqueness prevails in 
some important cases. They will also provide a better understanding of Proposition 2.

Corollary 2-A. When firms have chosen the same level of factor $z$ then $p_{1}^{*}=p_{2}^{*} \stackrel{\text { def }}{=} p^{*}$, $\bar{p}_{1}=\bar{p}_{2} \stackrel{\text { def }}{=} \bar{p}$ and the symmetric profile $\left(\min \left(p^{*}, \bar{p}\right), \min \left(p^{*}, \bar{p}\right)\right)$ is the unique payoffdominant pure strategy Nash equilibrium in the second stage of the game.

Corollary 2-B. When firms have not chosen the same level of factor $z$ and $\max \left(z_{1}, z_{2}\right) \geqslant$ $\tilde{z}$, then there exists $i \in\{1,2\}$ such that $\bar{p}_{i}=\min \left(p_{1}^{*}, p_{2}^{*}, \bar{p}_{1}, \bar{p}_{2}\right)$ and the symmetric profile $\left(\bar{p}_{i}, \bar{p}_{i}\right)$ is the unique payoff-dominant pure strategy Nash equilibrium at the second stage of the game.

Corollary 2-C. When firms have not chosen the same level of factor $z$ and $\max \left(z_{1}, z_{2}\right)<$ $\tilde{z}$ and $\bar{p}_{i} \neq \hat{p}_{j}$ then there exists $i \in\{1,2\}$ such that $p_{i}^{*}=\min \left(p_{1}^{*}, p_{2}^{*}, \bar{p}_{1}, \bar{p}_{2}\right)$ and the interval I is an non degenerated continuum of payoff-dominant pure strategy Nash equilibria in the second stage of the game.

Corollary 2-D. When $\bar{p}_{i}=\hat{p}_{j}$ then the symmetric profile $\left(\bar{p}_{i}, \bar{p}_{i}\right)$ ) is the unique (payoffdominant) pure strategy Nash equilibrium in the second stage of the game.

The following Table presents the results of the price competition in the different configurations.

\begin{tabular}{|c|c|c|c|}
\hline & $\begin{array}{c}\min \left(\bar{p}_{1}, \bar{p}_{2}\right) \leqslant \min \left(p_{1}^{*}, p_{2}^{*}\right) \\
\Leftrightarrow \max \left(z_{1}, z_{2}\right) \geqslant \tilde{z}\end{array}$ & \multicolumn{2}{|c|}{$\begin{array}{c}\min \left(\bar{p}_{1}, \bar{p}_{2}\right)>\min \left(p_{1}^{*}, p_{2}^{*}\right) \\
\Leftrightarrow \max \left(z_{1}, z_{2}\right)<\tilde{z}\end{array}$} \\
\hline$z_{1}=z_{2}$ & $\begin{array}{c}2 \mathrm{~A} \\
\text { Stage 2: Uniqueness } \\
p^{P D N}=\bar{p}_{1}=\bar{p}_{2} \equiv \bar{p}\end{array}$ & \multicolumn{2}{|c|}{$\begin{array}{l}\text { Stage 2: Uniqueness } \\
p^{P D N}=p_{1}^{*}=p_{2}^{*} \equiv p^{*}\end{array}$} \\
\hline$z_{1} \neq z_{2}$ & $\begin{array}{c}2 \mathrm{~B} \\
\text { Stage 2: Uniqueness } \\
p^{P D N}=\min \left(\bar{p}_{1}, \bar{p}_{2}\right)\end{array}$ & $\begin{array}{c}2 \mathrm{C}\left(\text { if } \bar{p}_{i} \neq \hat{p}_{j}\right) \\
\text { Stage } 2: \text { Multiplicity } \\
\qquad p^{P D N} \in I\end{array}$ & $\begin{array}{c}2 \mathrm{D}\left(\text { if } \bar{p}_{i}=\hat{p}_{j}\right) \\
\text { Stage } 2: \text { Uniqueness } \\
p^{P D N}=\min \left(\bar{p}_{1}, \bar{p}_{2}\right)\end{array}$ \\
\hline
\end{tabular}

Table 1: The different cases of price competition. 
Figures 3 and 4 illustrate respectively Case $2 \mathrm{~B}$ and $2 \mathrm{C}$.

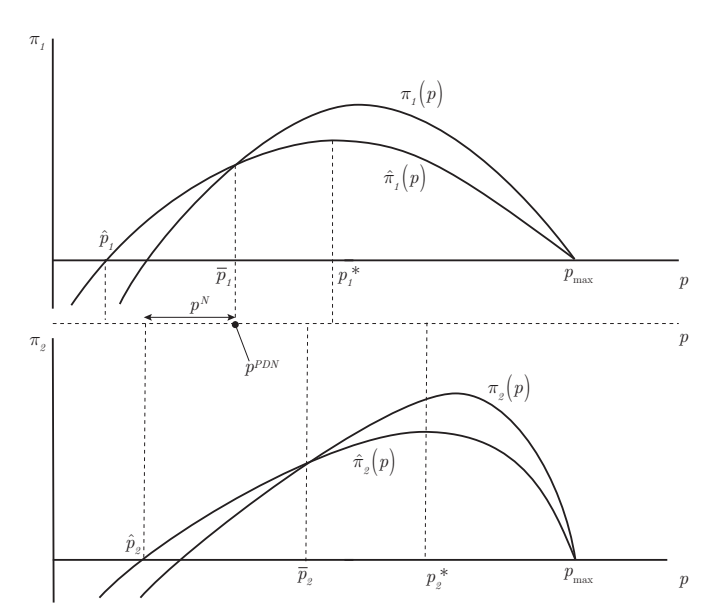

Figure 3: Case 2B

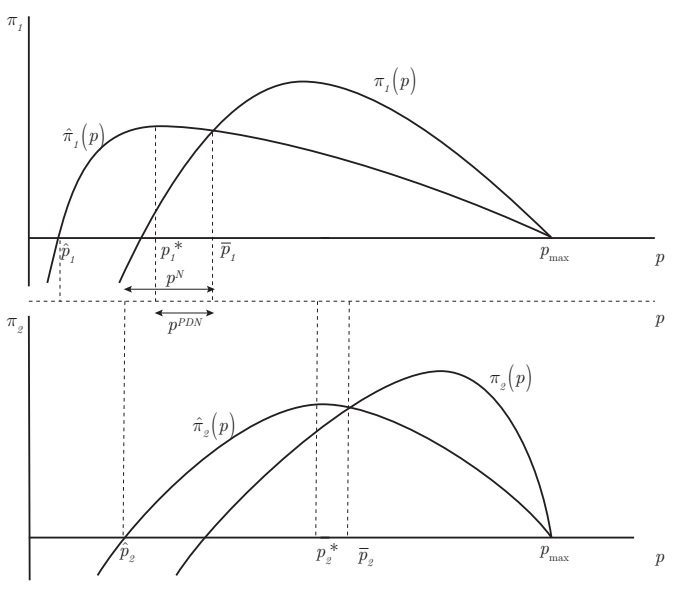

Figure 4: Case 2C

The payoff dominance criterion is insufficient to achieve the solution's uniqueness in the second stage. The corollary 2-C shows that the coordination problem (multiplicity of equilibria) follows from the profits geometry. But the corollary 2-A shows that the coordination problem may be solved in the endogenous way by the agents' decisions upstream, whatever the special values of the parameters.

\section{The first stage of the game}

In this section, firms determine their level of fixed factors anticipating the effect on the price equilibria at the second stage of the game. As we are in price competition, the profit function of the first stage of the game inherits the potential discontinuity of the profit function in the second stage of the game. Thus, for each outcome of the game, we will have to look for the consequences of unilateral deviation in $z$ on the profit function. Two problems may arise. First, we have to consider the potential asymmetry between the effect of a deviation in $z$ on the market price, i.e. it is the fixed factor level of the firm with the highest one that alone determines the market price. Secondly, a deviation in the level of the fixed factors can also modify the nature of the equilibrium 
because, as we have shown in Lemma 2, the relative position of $\bar{p}(z)$ and $p^{*}(z)$ depends on the position of $z$ according to $\tilde{z}$. So, before explicitly resolving the equilibrium in the first stage of the game, we have to go back to the geometry of the profit function.

\subsection{The geometry of the profit function, once again}

Let us define: $\Pi^{*}(z) \stackrel{\text { def }}{=} \hat{\pi}(\bar{p}(z), z)$ and $\Pi^{* *}(z) \stackrel{\text { def }}{=} \hat{\pi}\left(p^{*}(z), z\right)$. We define, $z^{*}=\arg \max \left\{\Pi^{*}(z)\right\}$ and $z^{* *}=\arg \max \left\{\Pi^{* *}(z)\right\}$. Finally, we define the function $\Pi(z)$ satisfying:

$$
\left\{\begin{array}{l}
\Pi(z)=\Pi^{* *}, \forall z \in(0, \tilde{z}] \\
\Pi(z)=\Pi^{*}, \forall z \in(\tilde{z},+\infty)
\end{array}\right.
$$

$\Pi(z)$ characterizes the profit function of both firm when they choose the same level of fixed factor. When $z \geqslant \tilde{z}$, it also characterizes the profit of the firm with the highest level of fixed factor.

Lemma 3 (Geometry of the profit function).

$$
\begin{aligned}
& \frac{d \Pi^{*}}{d z}(z)=\frac{\partial \hat{\pi}}{\partial p}(\bar{p}(z), z) \frac{d \bar{p}}{d z}(z)+\frac{\partial \hat{\pi}}{\partial z}(\bar{p}(z), z) \\
& \frac{d \Pi^{* *}}{d z}(z)=\frac{\partial \hat{\pi}}{\partial z}\left(p^{*}(z), z\right) \\
& \forall z \neq \tilde{z}, \Pi^{* *}(z)>\Pi^{*}(z) \\
& \Pi^{* *}(\tilde{z})=\Pi^{*}(\tilde{z}) \text { and } \frac{d \Pi^{* *}}{d z}(\tilde{z})=\frac{d \Pi^{*}}{d z}(\tilde{z})=\frac{\partial \hat{\pi}}{\partial z}\left(p^{*}(\tilde{z}), \tilde{z}\right)
\end{aligned}
$$

Proof: (6a) is obtained by taking the total differential of the definition. For (6b) and (6c), we use the fact that $p^{*}(z)$ maximise $\hat{\pi}(p, z)$ for a given $z$. (6d) is straightforward.

The relative position of $z^{*}$ and $z^{* *}$, on the one hand, with $\tilde{z}$, on the other hand, will depend on the property of the demand function and the value of the elasticity of the production according to the level of the fixed factor (the parameter $\alpha$ ). We 
can have two cases: $z^{*}>\tilde{z}$ (it will also hold for $z^{* *}$ ) or $z^{*} \leqslant \tilde{z}$. Both cases are possible and are illustrated respectively in Figures 6-a and 6-b. For example, with a linear demand function, case 6 -a occurs when $\alpha$ is low. Those figures also illustrate the general property enunciated in Lemma 3.

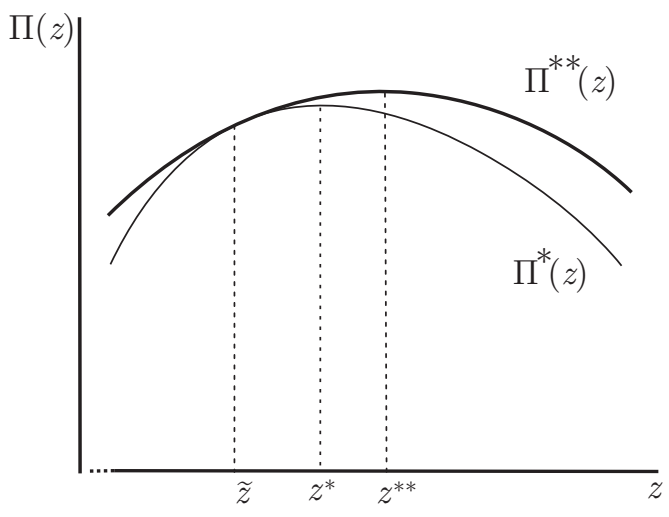

Figure 5-a: $z^{*}>\widetilde{z}$

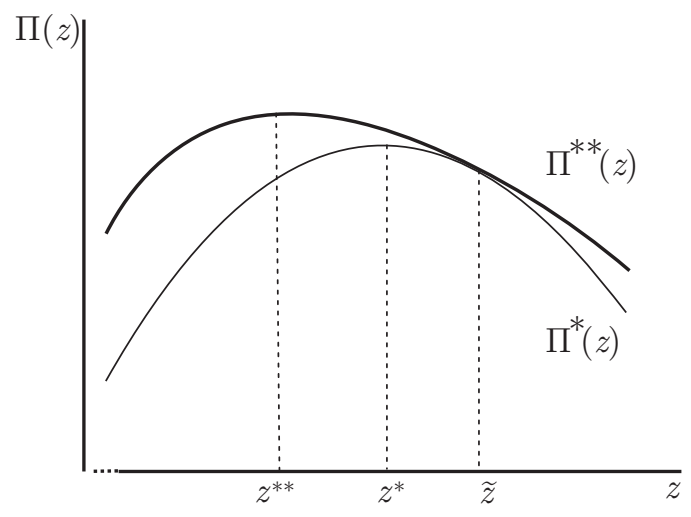

Figure 5-b: $z^{*} \leqslant \widetilde{z}$

We can now solve the first stage of the game.

\subsection{Equilibrium prediction for the first stage game}

The cases 5-a and 5-b determine the equilibrium prediction of the first stage game.

Proposition 3. There exists a unique payoff dominant pure strategy Nash equilibrium in the first-stage of the game.

i) If $z^{*}>\tilde{z}$ (Fig. 6-a), there exists an infinity of pure strategy Nash equilibria in the first-stage of the game, with $\left(z^{*}, z^{*}\right)$ the unique payoff dominant one.

ii) If $z^{*} \leqslant \tilde{z}$ (Fig. 6-b), there exists an infinity of pure strategy Nash equilibria in the first-stage of the game with $\left(z^{* *}, z^{* *}\right)$ the unique payoff dominant one.

Proof: in the appendix

Three points have to be noticed. First, the equilibrium prediction of the first stage is unique. Even if there is a multiplicity of Nash Equilibria in this setting, the payoff 
dominance criterion is sufficient to achieve uniqueness. Secondly, the equilibrium prediction of the first stage is symmetric i.e. the firms choose the same level of the fixed factors. It implies that the cases $2 \mathrm{~B}$ and $2 \mathrm{C}$ (Table 1 ) never occur in the second stage. The choice of the level of the fixed factor allows the firms to endogenously solve the problem of coordination in the second stage. When considering the whole game, we can now predict a unique outcome price in the second stage. The sequential choice of the production factors is thus, by itself, a powerful mean of coordination. However, and it will be our last point, the qualitative properties of the equilibrium prediction for the whole game depend on the geometry of the profit function (the relative position of $z^{*}$ and $\tilde{z}$ ). As we have shown, this geometry is determined by the shape of the demand function and the convexity of the short-term cost function, captured by the parameter $\alpha$. Proposition 3 distinguishes two cases. For a given demand function and for a given $\alpha$ (possibly low), both firms choose $z^{*}$ in the first stage and quote $\bar{p}\left(z^{*}\right)$. For other values of $\alpha$ (possibly high), both firms choose $z^{* *}$ in the first stage and quote $p^{*}\left(z^{* *}\right)$. Thus, it appears that firms coordinate on a "high price". Let us now examine if this price corresponds to a collusive one.

\subsection{The collusive nature of the equilibrium}

We define the collusive outcome as the solution of the joint-profit maximization program of two firms equally sharing the production. Because it is impossible for firms to legally enforce an explicit agreement, we add a constraint of non-profitable deviation. This program can be written:

$$
\left\{\begin{array}{c}
\max _{p, z} 2 \hat{\pi}(p, z) \\
\text { s.t. } p \leq \bar{p}(z)
\end{array}\right.
$$

As a simple application of the envelope theorem, and because of the geometry of the profit function, the results are as follows. When the constraint is binding, the solution 
of joint-profit maximization problem is $\left(z^{*}, \bar{p}\left(z^{*}\right)\right)$, which corresponds to a second best from the point of view of the firms. When the constraint is slack, $\left(z^{* *}, p^{*}\left(z^{* *}\right)\right)$ is the solution, corresponding to the cartel capacity and price.

Thus, the unique equilibrium prediction of the whole game corresponds to a collusive outcome. Our model provides a setting in which tacit collusion occurs in the sense of Ivaldi et al. (2003), and this result is obtain in a non-repeated two-stage game interaction. It is not the possibility of retaliation that drives the existence of tacit collusion.

Dastidar (2001) has discussed the possibility for the collusive outcome to be a Nash equilibrium of Bertand competition in a single-shot game. This paper goes further. Not only because we prove that our solution is a subgame perfect Nash equilibrium, but also because, combining a clear mechanism of equilibrium selection with a sequential choice of production factors with soft capacity constraint, we show that tacit collusion is the only predictable outcome of the whole game.

\section{Conclusion}

Our objective was to provide an example of a market in which tacit collusion can be obtained as a predictable result of a non-repeated framework. To do this, we have built a model with assumptions very close to the canonical textbook model of Bertrand competition: price competition, non-repeated interaction, homogeneity, constant returns to scale. Our only departure from this setting is that we rely on a two-factor production function chosen sequentially.

The conventional wisdom, based on Bertrand's original result, is to believe that price competition is a much more drastic form of competition than quantity. Even if there are few firms in the market, Bertrand's result shows that some form of imperfect competition can lead to marginal cost pricing and thus to social optimality. Since Edgeworth (1925), there has been a long-standing debate on this statement. By bridging 
two important lines of this literature distinguished by Vives (1999), the BertrandEdgeworth approach with capacity constraints, and that of convex costs (Dastidar, 1995), our paper is also a contribution to the resolution of the Bertrand Paradox.

An interesting property of our approach with a two-factor production function is that it disentangles any assumption about returns to scale from the convexity of marginal cost in the second stage. A natural extension of this paper would be to consider a more general form of the production function with possibly various types of returns-to-scale. This generalization would certainly be the proper framework to study the effect of the number of firms at the equilibrium and particularly the way this interacts with the mechanism of coordination in the first stage. Dastidar (2001) has shown that, when costs are convex, the effect of the number of firms can be very counter-intuitive, because the cost penalty incurred by a firm unilaterally lowering its price is increased when more firms operate in the market. Obviously, in our two-stage approach, the number of firms will also have an influence on the equilibrium value of the fixed factor, and thus on the geometry of the profit function. Those effects will not necessarily be trivial, especially if we consider various production functions and various returns to scale. A related point is the influence of fixed costs on the equilibrium's existence as discussed recently by Saporiti and Coloma (2010), Dastidar (2011a) and Dastidar (2011b). In our model, the cost of the fixed factor can be interpreted as a fixed cost in the second stage. But, because this cost is sunk, it will have no effect on the existence of the equilibrium.

\section{References}

Abbink, K. and J. Brandts (2008). 24. pricing in Bertrand competition with increasing marginal costs. Games and Economic Behavior 63(1), 1 - 31 .

Abreu, D. (1986). Extremal equilibria of oligopolistic supergames. Journal of Economic Theory 39(1), $191-225$. 
Allen, B., R. Deneckere, T. Faith, and D. Kovenock (2000). Capacity precommitment as a barrier to entry: A Bertrand-Edgeworth approach. Economic Theory 15, 501530.

Allen, B. and M. Hellwig (1986). Bertrand-Edgeworth oligopoly in large markets. Review of Economic Studies 53, 175-204.

Aumann, R. J. (1976). Agreeing to disagree. The Annals of Statistics 4(6), pp. 12361239.

Bagh, A. (2010). Pure strategy equilibria in bertrand games with discontinuous demand and asymmetric tie-breaking rules. Economics letters 108, 277-279.

Baye, M. R. and J. Morgan (1999). A folk theorem for one-shot bertrand games. Economics Letters 65(1), $59-65$.

Baye, M. R. and J. Morgan (2002). Winner-take-all price competition. Economic Theory 19(2), $271-282$.

Benoit, J.-P. and V. Krishna (1987). Dynamic duopoly: Prices and quantities. Review of Economic Studies 54(1), 23 - 35.

Chowdhury, P. R. (2009). Bertrand competition with non-rigid capacity constraints. Economics Letters 103(1), 55 - 58.

Cooper, Russell, W., D. V. DeJong, R. Forsythe, and T. W. Ross (1990). Selection criteria in coordination games: Some experimental results. American Economic Review 80, 218-233.

Dastidar, K. G. (1995). On the existence of pure strategy Bertrand equilibrium. Economic Theory 5, 19-32.

Dastidar, K. G. (2001). Collusive outcomes in price competition. Journal of Economics 73(1), 81-93. 
Dastidar, K. G. (2011a). Bertrand equilibrium with subadditive costs. Economics Letters 112, 202-204.

Dastidar, K. G. (2011b). Existence of Bertrand equilibrium revisited. International Journal of Economic Theory 7, 331-350.

Davidson, C. and R. Deneckere (1986). Long-term competition in capacity, shortrun competition in price, and the cournot model. Rand Journal of Economics 17, $404-415$.

Dixon, H. (1986). The Cournot and SBertrand outcomes as equilibria in a strategic metagame. Economic Journal 96(384), 59 - 70.

Edgeworth, F. (1925). The pure theory of monopoly. Papers Relating to Political Economy 1, 111-42.

Feuerstein, S. (2005). Collusion in industrial economics a survey. Journal of Industry, Competition and Trade 5, 163-198.

Friedman, J. W. (1971). A noncooperative equilibrium for supergame. Review of Economic Studies 38, 1-12.

Harsanyi, J. C. and R. Selten (1988). A General Theory of Equilibrium Selection in Games. Cambridge MA: The MIT Press.

Hoernig, S. H. (2002). Mixed Bertrand equilibria under decreasing returns to scale: an embarrassement of riches. Economic Letters 74, $359-362$.

Hoernig, S. H. (2007). Bertrand games and sharing rules. Economic Theory $31(3)$, $573-585$.

Ivaldi, M., B. Jullien, P. Rey, P. Seabright, and J. Tirole (2003). The economics of tacit collusion. Final Report for DG Competition, European Commission. 
Kaplan, T. R. and D. Wettstein (2000). The possibility of mixed-strategy equilibria with constant-returns-to-scale technology under Bertrand competition. Spanish Economic Review 2, 65-71.

Kreps, D. and J. Scheinkman (1983). Quantity precommitment and Bertrand competition yield Cournot outcomes. Bell Journal of Economics Bulletin 13, 111-122.

Novshek, W. and P. R. Chowdhury (2003). Bertrand equilibria with entry: limit results. International Journal of Industrial Organization 21(6), 795 - 808.

Saporiti, A. and G. Coloma (2010). Bertrand competition in markets with fixed costs. The BE Journal of Theoretical Economics 10(1), 27.

Vives, X. (1980). Rationing and Bertrand-Edgeworth equilibria in large markets. Economics Letters 27, 113-116.

Vives, X. (1999). Oligopoly Pricing, old ideas and new tools. Cambridge MA: The MIT Press.

\section{Appendix 1: Proof of Lemma 2}

Let us remark that $p^{*}$ solves $\hat{\pi}^{\prime}(p)=0$. When $\bar{p}(z)=p^{*}(z) \stackrel{\text { def }}{=} \tilde{p}, \hat{\pi}^{\prime}(\bar{p})=0$. After calculations, we get the right part of (5-a). Due to the strict concavity of $\hat{\pi}$ according to $p$ (assumption (4)), we have $: \bar{p}(z)>p^{*}(z) \Leftrightarrow \hat{\pi}^{\prime}(\bar{p})<0$. After some easy calculations, we then get the right part of $(5 b)$.

Let's now prove the uniqueness of $\tilde{p}$ and $\tilde{z}$. The left hand side of the expression $\frac{1}{1-\alpha} \frac{1}{2^{\frac{1}{1-\alpha}}-1}=1+\frac{1}{\mathcal{E}(p)}$ is a constant between 0 and 1 . For $p \in\left(0, p_{\max }\right)$, the right hand side is strictly increasing, taking values between $-\infty$ and 1 . That proves the uniqueness of $\tilde{p}$. Because $\bar{p}(z)$ is strictly decreasing on its definition set, the uniqueness of $\tilde{p}$ implies the uniqueness of $\tilde{z}$. 


\section{Appendix 2: Proof of Proposition 1}

When we consider price competition, we can no longer resolve the game using the reaction functions, because they are strongly discontinuous. Thus, we have to check for each possible strategy profile whether it is a Nash equilibrium or not. Let's first investigate symmetric strategy profiles belonging to the interval. When the competitor charges any price $p \in\left[\hat{p}_{i} ; \bar{p}_{i}\right]$, the best response for the firm $i$ is to quote the same price. When the firm $i$ quotes the same price, it gets $\hat{\pi}_{i}(p)$. We know that for all $p \geqslant \hat{p}_{i}$, $\hat{\pi}_{i}(p) \geqslant-T F C$ (see eq. (4b)). If the firm deviates (by quoting $p-\mu$ ), it gets $\pi_{i}(p-\mu)$. We also know that for all $p \in\left[\hat{p}_{i} ; \bar{p}_{i}\right], \hat{\pi}_{i}(p) \geqslant \pi_{i}(p)>\pi_{i}(p-\mu)$ (from eq (4c)). Since the firm must supply all the demand it faces, the increase in additional revenue (from higher sales) is less than the increase in costs: the firm must sell additional units at excessive marginal costs. By quoting $p+\mu$, the firm $i$ obtains no demand and gets zero variable profit. Hence it is optimal for each firm to quote the same price. There are no incentives to deviate, which proves the implication in Proposition 1. It also proves that all asymmetrical strategy profiles with at least one firm quoting a price in the interval are not Nash equilibria. We now have to investigate all the other strategy profiles, symmetric and asymmetric, in which none of the firms quote a price within the interval. It is easy to check that for all symmetric or asymmetric strategy profiles such that $p<\hat{p}$, the firm has interest to increase its price. The firm has interest to lower its price for symmetric profiles with $p>\bar{p}$. Finally, for asymmetric profiles with $p>\bar{p}$, the firm with the highest price will improve its profit in matching the other's price. Thus, asymmetric strategy profiles cannot be Nash Equilibria.

\section{Appendix 3: Proof of Proposition 2}

As shown in Proposition 1, all the Nash equilibria of the second stage are symmetric. So we can, without loss of generality, consider only symmetric strategy profiles $(p, p)$ and the associated gains for both firms $\left(\hat{\pi}_{1}(p), \hat{\pi}_{2}(p)\right)$. 
If we consider an open interval $\left(p_{a}, p_{b}\right)$ such that, $\forall p \in\left(p_{a}, p_{b}\right), \forall i \in\{1,2\}, \partial \hat{\pi}_{i}(p) / \partial p>$ 0 , then the symmetric strategy profile $\left(p_{b}, p_{b}\right)$ dominates all other profiles corresponding to a price in the interval. Considering the geometry of the profit function, the biggest open price interval with the profit of both firms being strictly increasing is $\left(0, \min \left(p_{1}^{*}, p_{2}^{*}\right)\right)$. If we restrict ourselves to the set of Nash equilibria the biggest such open interval is: $I=\left(0,\left(\min \left(p_{1}^{*}, p_{2}^{*}\right)\right)\right) \bigcap\left\{\left(\hat{p}_{1}, \bar{p}_{1}\right) \cap\left(\hat{p}_{2}, \bar{p}_{2}\right)\right\}$

$$
\begin{aligned}
& \text { If } \max \left(\hat{p}_{1}, \hat{p}_{2}\right) \geq \min \left(p_{1}^{*}, p_{2}^{*}, \bar{p}_{1}, \bar{p}_{2}\right) \text { then } I=\emptyset \text {. } \\
& \text { If } \max \left(\hat{p}_{1}, \hat{p}_{2}\right)<\min \left(p_{1}^{*}, p_{2}^{*}, \bar{p}_{1}, \bar{p}_{2}\right) \text { then } I=\left(\max \left(\hat{p}_{1}, \hat{p}_{2}\right), \min \left(p_{1}^{*}, p_{2}^{*}, \bar{p}_{1}, \bar{p}_{2}\right)\right)
\end{aligned}
$$

Thus, all symmetric profiles $(p, p)$ such that $p<\max \left(\hat{p}_{1}, \hat{p}_{2}, \min \left(p_{1}^{*}, p_{2}^{*}, \bar{p}_{1}, \bar{p}_{2}\right)\right)$ cannot be payoff-dominant pure strategy Nash equilibria.

Reasoning the same way, we can define the biggest open price interval with the profit of both firms being strictly decreasing and such that the symmetric strategy profiles corresponding to those prices are Nash equilibria, $\left(\min \left(\max \left(p_{1}^{*}, p_{2}^{*}\right), \bar{p}_{1}, \bar{p}_{2}\right), \min \left(\bar{p}_{1}, \bar{p}_{2}\right)\right)$. Thus all symmetric profiles $(p, p)$ such that $p>\min \left(\max \left(p_{1}^{*}, p_{2}^{*}\right), \bar{p}_{1}, \bar{p}_{2}\right)$ cannot be payoff-dominant pure strategy Nash equilibria.

Finally, over the closed interval $\left[\max \left(\hat{p}_{1}, \hat{p}_{2}, \min \left(p_{1}^{*}, p_{2}^{*}, \bar{p}_{1}, \bar{p}_{2}\right)\right), \min \left(\max \left(p_{1}^{*}, p_{2}^{*}\right), \bar{p}_{1}, \bar{p}_{2}\right)\right]$ all symmetric profiles corresponding to those prices are Nash equilibria, with the profit of both firms varying in the opposite way.

\section{Appendix 4: Proof of Proposition 3}

For a purpose of readability, we will denote by the subscript $h$ the firm with the highest level of fixed factor, by the subscript $l$ the one with the lowest level (i.e. $\left.z_{h}=\max \left(z_{1}, z_{2}\right) \geqslant z_{l}=\min \left(z_{1}, z_{2}\right)\right)$. Let's start by demonstrating Proposition 3 i).

Step 1: Asymmetric strategy profiles cannot be Nash equilibria of the first stage game. a) If we are in the case $\left(z_{l}<z_{h}<\tilde{z}\right)$ then the equilibria of the second stage are characterized by the Corollary 2-C. Firms have a coordination problem. They are not 
able to predict the price in the second stage. But, by choosing to match the other firm's fixed factor level, they can solve endogenously the coordination problem. So, for asymmetric profiles of that kind, firms have an incentive to deviate. Those strategy profiles cannot be Nash equilibria of the first stage game. b) If we are in the case $\left(\tilde{z} \leqslant z_{h} \neq z^{*}\right)$, the equilibrium of the second stage is characterized by the Corollary 2-B. The profit of the firm $h$ is characterized by the function $\Pi^{*}\left(z_{h}\right)$. Thus, the firm $h$ has an incentive to deviate getting closer to $z^{*}$, the fixed factor level that maximises $\Pi^{*}(z)$. Those strategy profiles cannot be Nash equilibria of the first stage game. c) If we are in the case $\left(z_{l}<z_{h}=z^{*}\right)$, the firm $h$ has no incentive to deviate. Because of the definition of $p^{*}$, we have $\partial \hat{\pi}\left(p^{*}(z), z\right) / \partial p=0$. Because $z^{*} \geqslant \tilde{z}$, we have $p^{*}\left(z^{*}\right) \geqslant \bar{p}\left(z^{*}\right)$ and thus because $\partial^{2} \hat{\pi}(p, z) / \partial p^{2}<0$ we have $\partial \hat{\pi}\left(\bar{p}\left(z^{*}\right), z^{*}\right) / \partial p>\partial \hat{\pi}\left(p^{*}\left(z^{*}\right), z^{*}\right) / \partial p=0$. According to the definition of $z^{*}$, we have $d \Pi\left(z^{*}\right) / d z=0$. Using equation (6b), and the fact that $d \bar{p}(z) / d z<0$, we deduce that $\partial \hat{\pi}\left(\bar{p}\left(z^{*}\right), z^{*}\right) / \partial z>0$. Finally, because $z_{l}<z^{*}$ and $\partial^{2} \hat{\pi}(p, z) / \partial z^{2}<0$, we have $\partial \hat{\pi}\left(\bar{p}\left(z^{*}\right), z_{l}\right) / \partial z>0$. This last expression characterizes the effect of an increase in $z$ on the profit of $l$ (i.e. there is no price effect, only the cost effect). It implies that the firm $l$ has always an incentive to deviate by increasing its level of fixed factor. Those strategy profiles cannot be Nash equilibria of the first stage game. a), b) and c) characterize all the possible asymmetric profiles of the first stage game and proves step 1.

Step 2: $\left(z^{*}, z^{*}\right)$ is a Nash Equilibrium of the first stage game.

If a firm unilaterally decide to increase its level of fixed factor to $z_{+}>z^{*}$, it will become the only firm with the high level of fixed factor. Its profit will be $\Pi^{*}\left(z_{+}\right)$. But, because $\Pi^{*}(z)$ reach it's maximum in $z^{*}$, we necessarily have $\Pi^{*}\left(z^{*}\right)>\Pi^{*}\left(z_{+}\right)$. Firms have no interest to unilaterally increase the level of the fixed factor. If a firm unilaterally decides to decrease its level of fixed factor to $z_{-}<z^{*}$, its profit will be $\hat{\pi}\left(\bar{p}\left(z^{*}\right), z_{-}\right)$. We have demonstrate in step 1c that $\forall z<z^{*}, \partial \hat{\pi}\left(\bar{p}\left(z^{*}\right), z\right) / \partial z>0$. Thus, we have $\hat{\pi}\left(\bar{p}\left(z^{*}\right), z_{-}\right)<\hat{\pi}\left(\bar{p}\left(z^{*}\right), z^{*}\right)$. That proves step 2. Firms have no interest to unilaterally decrease the level of the fixed factor. That ends the proof of step 2 . 
Step 3: $\left(z^{*}, z^{*}\right)$ dominates all the other symmetric strategy profiles of the first stage game.

With the same reasoning as in the preceding steps, it can easily be demonstrated that for "small" $\epsilon,\left(z^{*}+\epsilon, z^{*}+\epsilon\right)$ are also Nash equilibria of the game. But because all the Nash equilibria belong to the set of the symmetric strategy profiles and, because, in the symmetric cases, the firms earn $\Pi(z)$, when $z^{*}>\tilde{z}, z^{*}$ maximise $\Pi(z)$ and thus $\left(z^{*}, z^{*}\right)$ is payoff dominant.

Let's turn now to Proposition 3 ii).

Step 1': Asymmetric strategy profiles cannot be Nash equilibria of the first stage game. Case a) If we are in the case $\left(z_{l}<z_{h}<\tilde{z}\right)$, the reasoning is exactly same as in step 1 a). b) If we are in the case $\left(\tilde{z}<z_{h}\right)$, the equilibrium of the second stage is characterized by the Corollary 2-B. The profit of the firm $h$ is characterized by the function $\Pi^{*}\left(z_{h}\right)$. Thus, the firm $h$ has an incentive to deviate getting closer to $\tilde{z}\left(\Pi^{*}(z)\right.$ is strictly decreasing for $\left.z>\tilde{z}\right)$. Those profiles cannot be Nash equilibria of the first stage game. c) If we are in the case $\left(z_{l}<z_{h}=\tilde{z}\right)$. Let's define $z^{\#}=\arg \max \{\hat{\pi}(\bar{p}(\tilde{z}), z)\}$, the optimal level of the fixed factor for the firm $l$,when $z_{h}=\tilde{z}$. Because of the concavity according to $z$ of the function $\hat{\pi}$ and the fact that, when $z^{*}<\tilde{z}, \partial \hat{\pi}(\bar{p}(\tilde{z}), \tilde{z}) / \partial z<0$, we have $z^{\#}<\tilde{z}$. We can distinguish two subcases. c1) If $z_{l} \neq z^{\#}$, then the firm $l$ has an incentive to deviate, choosing $z^{\#}$. c2) If $z_{l}=z^{\#}$, we can show that the firm $h$ has an incentive to deviate, choosing $z^{\#}$, because $\Pi^{* *}\left(z^{\#}\right)>\Pi^{* *}(\tilde{z})=\Pi^{*}(\tilde{z})$. For proving that, we have to notice that by definition of $z^{\#}$, $\partial \hat{\pi}\left(\bar{p}(\tilde{z}), z^{\#}\right) / \partial z=0 . z^{\#}<\tilde{z} \Rightarrow p^{*}\left(z^{\#}\right)>p^{*}(\tilde{z})$, and thus because $\partial^{2} \hat{\pi}(p, z) / \partial p \partial z<0$, we get $\partial \hat{\pi}\left(p^{*}\left(z^{\#}\right), z^{\#}\right) / \partial z<\partial \hat{\pi}\left(p^{*}(\tilde{z}), z^{\#}\right) / \partial z=\partial \hat{\pi}\left(\bar{p}(\tilde{z}), z^{\#}\right) / \partial z=0$. It implies that, due to the strict concavity of the profit function $\Pi^{* *}$ is strictly decreasing between $z^{\#}$ and $\tilde{z}$ and thus, $\Pi^{* *}\left(z^{\#}\right)>\Pi^{* *}(\tilde{z})$. Those profiles cannot be Nash equilibria of the first stage game. a), b) c1) and c2) characterize all the possible asymmetric profiles of the first stage game and proves step 1' 7 .

Step 2': $\left(z^{* *}, z^{* *}\right)$ is a Nash Equilibrium of the first stage game. 
If one of the two firms choose to unilaterally deviate, two cases are possible. If it chooses $z<\tilde{z}$, the equilibria of the second stage are characterized by the Corollary 2-C. We go back to the coordination problem and the firm has no incentive to do that. If the firm chooses $z \geqslant \tilde{z}$, it will become the only firm with the high level of fixed factor. Its profit will be $\Pi(z)$. But, because $\Pi(z)$ reaches its maximum in $z^{* *}$, the firm has no incentive to do that.

Step 3': $\left(z^{* *}, z^{* *}\right)$ dominates all the other symmetric strategy profiles of the first stage game.

If we define $\tilde{z}^{-}$such that $\tilde{z}^{-}<z^{* *}$ and $\Pi\left(\tilde{z}^{-}\right)=\Pi(\tilde{z})$, then all symmetric profiles $(z, z)$ such that $z \in\left[\tilde{z}^{-}, \tilde{z}\right]$ are Nash equilibria of the game. In those cases, the firms have no incentive to unilaterally deviate choosing $z^{\prime}<\tilde{z}$, because the equilibrium of the second stage is characterized by corollary 2-C, and we are back to the coordination problem. Firms have also no incentive to unilaterally deviate choosing $z^{\prime} \geqslant \tilde{z}$ because $\Pi(z) \geqslant \Pi\left(z^{\prime}\right)$.

Because all the Nash Equilibria belongs to the set of symmetric strategy profiles, $\left(z^{* *}, z^{* *}\right)$ is the only payoff dominant Nash Equilibrium of the game. 


\section{Notes}

${ }^{1}$ See Vives (1999), section 5.1, pp 117-123.

${ }^{2}$ Other extensions with mixed-strategy equilibria and positive profit levels are provided by Baye and Morgan (1999), Kaplan and Wettstein (2000) and Hoernig (2002).

${ }^{3}$ In the sense of Aumann (1976), cf Harsanyi and Selten (1988) p. 359. There is a huge literature on equilibrium selection in games, see Cooper et al. (1990), for an introduction.

${ }^{4}$ For simplicity, we will use a Cobb-Douglas production function, but what determines our results is the convexity of the short term marginal cost which depends on the decreasing marginal productivity of the variable factor, a universal assumption in economics. Our results do not depend on the specification of the production function.

${ }^{5}$ Our model provides foundations for the notion of non-rigid capacity constraint introduced by Chowdhury (2009) directly in the cost function.

${ }^{6}$ Dixon (1986) considers the same structure for the production function (case b). However the price setting in the second stage differs from ours.

${ }^{7}$ For a specific value of $\alpha$, we have $z^{*}=z^{* *}=z^{\#}=\tilde{z}$. In this case $z_{l}=z^{\#}=\tilde{z}=z_{h}$ is not an asymmetric outcome. 